\title{
TOlerÂnCIA de Cultivares de ARRoz IRRIGAdo (Oryza sativa) AO Herbicida Nicosulfuron e À Mistura Formulada de IMAZETHAPYR + IMAZAPIC ${ }^{1}$
}

\author{
Tolerance of Rice (Oryza sativa) cultivars to Nicosulfuron and to the Formulated Mixture \\ Imazethapyr + Imazapic
}

\author{
FONTANA, L.C. ${ }^{2}$, AGOSTINETTO, D. ${ }^{3}$, PINTO, J.J.O. ${ }^{4}$, RIGOLI, R.P. ${ }^{5}$, FIGUEREDO, S.S. ${ }^{2}$ e \\ ROSENTHAL, M.D. ${ }^{6}$
}

\begin{abstract}
RESUMO - O objetivo deste estudo foi avaliar a tolerância de cultivares de arroz irrigado ao herbicida nicosulfuron e à mistura formulada de imazethapyr + imazapic. Para isso, foram conduzidos experimentos em casa de vegetação da Faculdade de Agronomia Eliseu Maciel (UFPel), no município de Capão do Leão (RS). Os tratamentos constaram de dois cultivares de arroz irrigado (IRGA 417 e IRGA 422CL) e de sete doses dos herbicidas imazethapyr + imazapic e nicosulfuron, aplicados em arroz (estádios de desenvolvimento $V_{2}$ ou $V_{4}$ ). As variáveis analisadas foram fitotoxicidade dos herbicidas, estatura de planta, massa seca da parte aérea e área foliar. Houve interações significativas entre os fatores estudados para as variáveis-respostas avaliadas. Incrementos nas doses dos herbicidas resultaram em respostas crescentes de fitotoxicidade ao arroz, resultando em redução no crescimento das plantas. O cultivar IRGA 417 foi suscetivel à ação dos herbicidas. Por sua vez, a tolerância do cultivar IRGA 422CL não é restrita à mistura formulada de imazethapyr + imazapic, ocorrendo também para o herbicida nicosulfuron.
\end{abstract}

Palavras-chave: imidazolinona, sulfoniluréia, inibidores da ALS, IRGA 422CL, IRGA 417.

\begin{abstract}
The objective of this study was to evaluate the tolerance of rice cultivars to the formulated mixture of nicosulfuron and imazethapyr + imazapic. Greenhouse experiments were carried out at the Faculdade de Agronomia Eliseu Maciel (UFPel) in Capão do Leão (RS). The treatments consisted of two rice cultivars ('IRGA 417' and 'IRGA 422CL') and seven rates of the herbicides imazethapyr + imazapic and nicosulfuron, applied on rice (stages $V_{2}$ or $V_{4}$ ). The variables evaluated were herbicide injury, plant height, shoot dry biomass and leaf area. Significant interactions were found among the factors tested for the response-variables evaluated. Increased herbicide rates resulted in increased rice injury responses leading to decreases in plant height, biomass, and leaf area. 'IRGA 417' cultivar was susceptible to the herbicides. On the other hand, the tolerance of cultivar 'IRGA 422CL' was not restricted to the imazethapyr+imazapic formulated mixture, also occurring with nicosulfuron.
\end{abstract}

Keywords: ALS-inhibitors, imidazolinone, sulfonylurea, 'IRGA 422CL', 'IRGA 417'.

1 Recebido para publicação em 18.4.2007 e na forma revisada em 29.11.2007.

2 Enga -Agr $\stackrel{a}{\text {, }}$ mestranda em Fitossanidade, FAEM/UF Pel, Caixa Postal 354, $96010-900$, Capão do Leão/RS, $<$ lisicamponogara@gmail.com>; ${ }^{3}$ Eng $^{0}-$ Agr $^{0}$., Dr., Professor da FAEM/UFPel, bolsista do CNPq; ${ }^{4}$ Eng $^{\mathrm{e}}$-Agr ${ }^{\mathrm{e}}$., M.Sc., Professor da FAEM/UFPel; ${ }^{5}$ Bióloga, Mestranda em Fitossanidade, FAEM/UFPel; ${ }^{6}$ Eng $^{\mathrm{a}}$-Agr ${ }^{\mathrm{a}}$., Dr ${ }^{\mathrm{a}}$, FAEM/UFPel. 


\section{INTRODUÇÃO}

A produtividade das lavouras de arroz irrigado (Oryza sativa sp.) ainda está aquém do potencial dos cultivares devido a uso de sementes de baixa qualidade, semeadura fora da época recomendada, manejo inadequado do solo e da água, adubação insuficiente e, principalmente, controle deficiente de plantas daninhas. Dentre as espécies daninhas que infestam as áreas de produção destaca-se o arroz-vermelho (Oryza sp.), em razão das dificuldades existentes para efetuar seu controle.

A similaridade entre o arroz-vermelho e o arroz cultivado impedia, até recentemente, o controle seletivo dessa planta daninha com herbicidas. Contudo, o desenvolvimento de novos cultivares de arroz irrigado tolerantes a herbicidas pertencentes ao grupo das imidazolinonas possibilitou o controle do arroz-vermelho de forma seletiva (Steele et al., 2002).

Essa nova tecnologia, que associa a utilização de cultivares tolerantes à aplicação de herbicidas não-seletivos, está sendo difundida através do sistema denominado Clearfield ${ }^{\circledR} \mathrm{e}$ tem mostrado eficácia no controle de arrozvermelho superior a $97 \%$ em resultados de pesquisa obtidos no RS (Villa et al., 2006). Neste sistema é utilizado o herbicida Only ${ }^{\circledR}$, o qual é constituído da mistura formulada dos herbicidas imazethapyr $\left(75 \mathrm{~g} \mathrm{~L}^{-1}\right)$ e imazapic $\left(25 \mathrm{~g} \mathrm{~L}^{-1}\right)$, aplicado em pós-emergência para controle de arroz-vermelho (Sosbai, 2005). O herbicida imazethapyr + imazapic pertence ao grupo químico das imidazolinonas, e seu mecanismo de ação é a inibição da enzima acetolactato sintase (ALS) - primeira enzima da rota de sintese dos aminoácidos de cadeia ramificada, valina, leucina e isoleucina (Trezzi \& Vidal, 2001).

O primeiro cultivar de arroz irrigado disponibilizado comercialmente para o sistema Clearfield ${ }^{\circledR}$ no Brasil foi o IRGA 422CL. Este cultivar apresenta como característica principal a tolerância ao herbicida Only ${ }^{\circledR}$ e foi obtido por meio do retrocruzamento do cultivar IRGA 417 com a linhagem tolerante às imidazolinonas (Lopes et al., 2003). O cultivar IRGA 417, utilizado como genitor recorrente, destaca-se pela produtividade, qualidade de grão e boa adaptabilidade a todas as regiões orizícolas do RS (Sosbai, 2005).
No entanto, há relatos de que o arroz cultivado tolerante ao herbicida imazethapyr possui também tolerância a vários outros herbicidas inibidores da enzima ALS, como imazaquin, imazapyr, nicosulfuron, pyrithiobac e triasulfuron (Webster \& Masson, 2001). Dentre esses, nicosulfuron, imazaquin e imazapyr demonstraram ser seletivos ao arroz mutado e eficientes no controle de plantas daninhas.

O herbicida Sanson ${ }^{\circledR}$, que corresponde a $40 \mathrm{~g} \mathrm{~L}^{-1}$ de nicosulfuron, também pertence à classe dos inibidores da ALS, fazendo parte do grupo químico das sulfoniluréias. É utilizado na cultura do milho e possui ação sistêmica, sendo aplicado em pós-emergência e recomendado para controlar várias espécies de plantas daninhas (Andrei, 2005).

As hipóteses da pesquisa são de que a tolerância do cultivar IRGA 422CL não está restrita ao herbicida imazethapyr + imazapic, sendo dependente do estádio em que a aplicação é realizada, e de que os herbicidas inibidores da ALS poderão causar dano aos cultivares de arroz não derivados de linhagens mutadas.

O objetivo deste estudo foi avaliar a tolerância dos cultivares de arroz irrigado IRGA 417 e IRGA 422CL ao herbicida nicosulfuron e à mistura formulada de imazethapyr + imazapic, em aplicações realizadas nos estádios de desenvolvimento $\mathrm{V}_{2}$ ou $\mathrm{V}_{4}$.

\section{MATERIAL E MÉTODOS}

Foram conduzidos dois experimentos em casa de vegetação, na Faculdade de Agronomia Eliseu Maciel da Universidade Federal de Pelotas (UFPel), localizada no municipio de Capão do Leão, RS, na estação de crescimento 2005/06.

As unidades experimentais foram compostas por vaso plástico com capacidade para 1,2 L (primeiro experimento) ou oito litros (segundo experimento), preenchido com solo peneirado. Em cada vaso foram semeadas 12 ou 25 sementes de arroz; após a emergência, procedeuse ao desbaste, selecionando quatro ou cinco plântulas para o primeiro e o segundo experimento, respectivamente.

O solo utilizado é classificado como Planossolo Hidromórfico Eutrófico Solódico, pertencente à Unidade de Mapeamento Pelotas 
(Embrapa, 1999), e a adubação foi realizada conforme as recomendações para a cultura (Sosbai, 2005).

O delineamento experimental utilizado foi o de blocos casualizados, com quatro repetições. Os tratamentos foram arranjados em esquema fatorial, em que o fator A comparou cultivares de arroz irrigado (IRGA 417 ou IRGA 422CL), o fator B testou herbicidas (imazethapyr + imazapic ou nicosulfuron) e o fator $\mathrm{C}$ avaliou doses dos herbicidas correspondentes a 0,00 (testemunha sem aplicação), 0,25, 0,75, $1,00,1,25$ e $1,50 \mathrm{~L} \mathrm{ha}^{-1}$ de Sanson ${ }^{\circledR}\left(40 \mathrm{~g} \mathrm{~L}^{-1} \mathrm{de}\right.$ nicosulfuron) ou Only ${ }^{\circledR}\left(75+25 \mathrm{~g} \mathrm{~L}^{-1}\right.$ de imazethapyr + imazapic). Nos tratamentos com o herbicida imazethapyr + imazapic foi adicionado à calda de pulverização um adjuvante não-iônico (Dash $\left.\mathrm{HC}^{\circledR}\right)$, na proporção de $0,3 \%$ v/v.

No primeiro experimento, a aplicação foi efetuada sobre plantas de arroz irrigado em estádio de desenvolvimento $\mathrm{V}_{2}$ e, no segundo, sobre plantas em estádio $\mathrm{V}_{4}$ (Counce et al., 2000), com auxílio de pulverizador costal pressurizado a $\mathrm{CO}_{2}$, munido de bico 110.015 do tipo leque, regulado com pressão de $210 \mathrm{kPa}$, que proporcionou aplicação de $150 \mathrm{~L} \mathrm{ha}^{-1}$ de calda herbicida.

A irrigação foi feita de acordo com a necessidade da cultura, sendo mantida uma lâmina de água permanente a partir do terceiro dia após aplicação dos herbicidas.

As variáveis analisadas foram fitotoxicidade, estatura de planta, massa seca da parte aérea e área foliar. A fitotoxicidade foi observada visualmente por três avaliadores, aos 7 , 14, 21 e 28 dias após a aplicação dos tratamentos (DAT), utilizando escala de zero (0) a cem (100), em que zero representou ausência de sintomas e 100 a morte das plantas. A estatura de planta $(\mathrm{cm})$, avaliada aos 14,21 e 28 DAT com auxílio de uma régua graduada, foi obtida pela média entre as plantas da medida do colmo principal, desde a coroa até a extremidade da última folha com colar formado. A determinação da área foliar $\left(\mathrm{cm}^{2}\right)$ foi realizada aos 28 DAT, com auxílio de medidor de área foliar. Posteriormente, as plantas foram colocadas para secagem em estufa com circulação de ar a $65{ }^{\circ} \mathrm{C}$, até massa constante.

Os dados obtidos foram analisados quanto à sua homocedasticidade e, posteriormente, submetidos à análise da variância $(\mathrm{p} \leq 0,05)$; sendo esta significativa, os dados foram analisados por regressão.

\section{RESULTADOS E DISCUSSÃO}

Em todas as variáveis analisadas, em ambos os experimentos, verificou-se interação significativa entre os fatores estudados, demonstrando que os resultados obtidos dependeram do cultivar, do herbicida e da dose aplicada (Figuras 1 a 8 ).

A fitotoxicidade herbicida às plantas, em geral, foi superior quando se aplicou o herbicida imazethapyr + imazapic, comparativamente ao nicosulfuron (Figuras 1 e 2).

Para os herbicidas e doses testados, o cultivar IRGA 417 mostrou-se mais suscetivel aos efeitos tóxicos em relação ao cultivar mutado IRGA 422CL. Esses resultados podem ser explicados pelo fato de o cultivar IRGA 422CL ser derivado de uma linhagem mutada e possuir a característica de tolerância às imidazolinonas (imazethapyr + imazapic), sendo tolerante também ao grupo das sulfoniluréias (nicosulfuron), confirmando os resultados obtidos por Webster \& Masson (2001). A fitotoxicidade observada no cultivar IRGA 417 demonstrou sua suscetibilidade a esses herbicidas, como foi relatado em estudos de deriva simulada na cultura do arroz (Bond et al., 2006; Dal Magro, 2006; Kurtz \& Street, 2003).

A fitotoxicidade aumentou com o incremento na dose e de forma mais acentuada no cultivar IRGA 417, comparativamente a IRGA 422CL (Figuras 1 e 2). O efeito do incremento da dose herbicida resultando em aumento na fitotoxicidade foi reportado em outros estudos, tanto para cultivares de arroz tolerantes como para cultivares sensiveis (Bond et al., 2006; Dal Magro et al., 2006; Kurtz \& Street, 2003; Levy Jr. et al., 2006; Ottis et al., 2003; Steele et al., 2002).

Os niveis de fitotoxicidade mostram, no cultivar IRGA 422CL, que os efeitos tóxicos dos herbicidas evoluíram até a terceira avaliação (21 DAT), reduzindo posteriormente com o desenvolvimento da cultura (Figuras 3 e 4). Este cultivar, por ser tolerante aos herbicidas testados, mostrou capacidade de recuperação, como observado por Ottis et al. (2003). Entretanto, o 


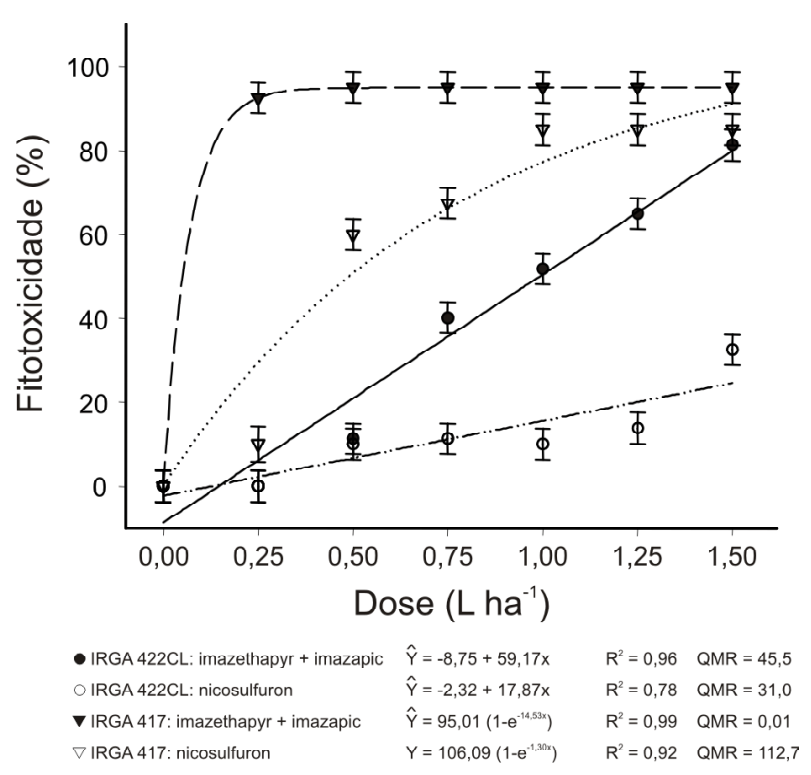

Figura 1 - Fitotoxicidade em cultivares de arroz irrigado, pela aplicação dos herbicidas Sanson ${ }^{\circledR}$ (40 $\mathrm{g} \mathrm{L}^{-1}$ de nicosulfuron) e Only ${ }^{\mathbb{B}}\left(75+25 \mathrm{~g} \mathrm{~L}^{-1}\right.$ de imazethapyr + imazapic), realizada no estádio $\mathrm{V}_{2}$, avaliada aos 21 dias após o tratamento. FAEM/UFPel, Capão do Leão/RS, 2005/06.

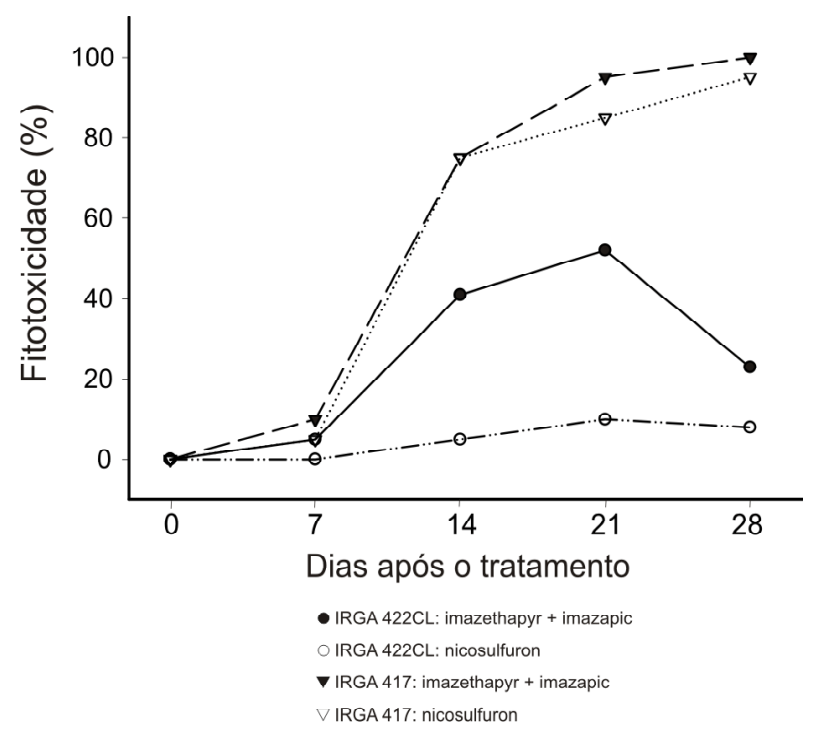

Figura 3 - Evolução da fitotoxicidade em cultivares de arroz irrigado, pela aplicação dos herbicidas $\operatorname{Sanson}^{\circledR}\left(40 \mathrm{~g} \mathrm{~L}^{-1} \mathrm{de}\right.$ nicosulfuron) e Only ${ }^{\circledR}\left(75+25 \mathrm{~g} \mathrm{~L}^{-1}\right.$ de imazethapyr + imazapic) na dose de $1 \mathrm{~L} \mathrm{ha}^{-1}$, no estádio $\mathrm{V}_{2}$. FAEM/UFPel, Capão do Leão/RS, 2005/06.

mesmo não ocorreu com o cultivar IRGA 417, pois a fitotoxicidade herbicida aumentou da primeira para a última época de avaliação, com evolução no efeito dos herbicidas, que resultou na morte das plantas.

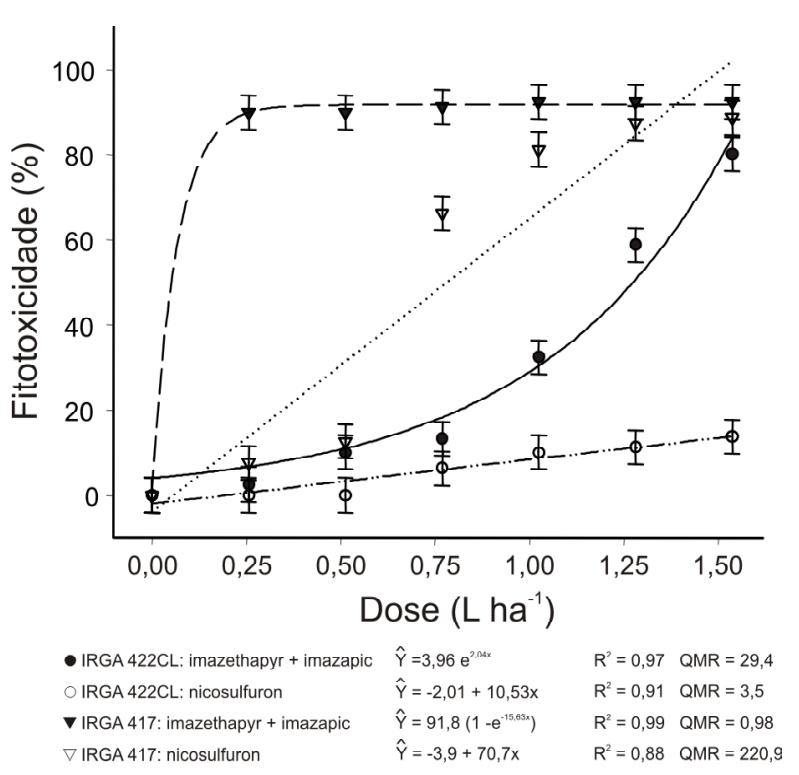

Figura 2 - Fitotoxicidade em cultivares de arroz irrigado, pela aplicação dos herbicidas Sanson ${ }^{\circledR}\left(40 \mathrm{~g} \mathrm{~L}^{-1}\right.$ de nicosulfuron) e Only ${ }^{\mathbb{B}}\left(75+25 \mathrm{~g} \mathrm{~L}^{-1}\right.$ de imazethapyr + imazapic), realizada no estádio $\mathrm{V}_{4}$, avaliada aos 21 dias após o tratamento. FAEM/UFPel, Capão do Leão/RS, 2005/06.

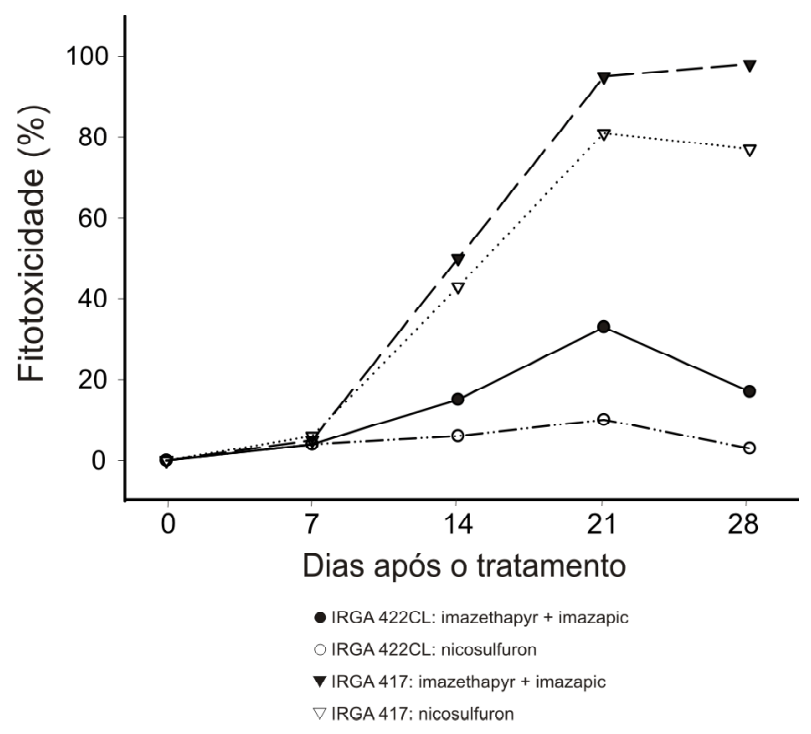

Figura 4 - Evolução da fitotoxicidade em cultivares de arroz irrigado, pela aplicação dos herbicidas $\operatorname{Sanson}^{\mathbb{R}}\left(40 \mathrm{~g} \mathrm{~L}^{-1} \mathrm{de}\right.$ nicosulfuron) e Only ${ }^{\circledR}\left(75+25 \mathrm{~g} \mathrm{~L}^{-1}\right.$ de imazethapyr + imazapic) na dose de $1 \mathrm{~L} \mathrm{ha}^{-1}$, no estádio $\mathrm{V}_{4}$. FAEM/UFPel, Capão do Leão/RS, 2005/06.

Outros estudos reportaram a habilidade de cultivares tolerantes em se recuperar dos efeitos negativos de nicosulfuron (Webster \& Masson, 2001) e imazethapyr (Levy Jr. et al., 2006; Steele et al., 2002; Villa et al., 2006). 
A estatura das plantas foi reduzida com o incremento da dose dos herbicidas em ambos os experimentos (Figuras 5 e 6). Essa redução foi mais evidente no cultivar IRGA 417, onde as plantas praticamente não cresceram após aplicação dos herbicidas, devido à paralisação do metabolis mo celular. Já o cultivar IRGA 422CL mostrou recuperação na estatura das plantas. Para ambos os cultivares, e nas doses testadas, o herbicida nicosulfuron demonstrou ser mais seletivo às plantas de arroz que imazethapyr + imazapic.

A recuperação do cultivar IRGA 422CL durante as épocas de avaliação ocorreu mesmo nos tratamentos em que se observou maior fitotoxicidade, exceto nas duas maiores doses de imazethapyr + imazapic (Figuras 5 e 6). Essa capacidade de recuperação se deve à tolerância do cultivar IRGA 422CL aos herbicidas estudados, que depende da dose utilizada. Para o cultivar IRGA 417 foi observada recuperação somente na menor dose do herbicida nicosulfuron, pois os demais tratamentos não permitiram o crescimento das plantas.

Segundo Webster \& Masson (2001), os acréscimos nas doses de imazethapyr podem ser prejudiciais ao cultivar tolerante, porém o crescimento das plantas ilustra a habilidade do arroz em se recuperar das injúrias observadas após a aplicação; a estatura de plantas tolerantes, observada no momento da colheita, pode não ser afetada pela aplicação de imazethapyr ou imazethapyr + imazapic (Pellerin \& Webster, 2004; Villa et al., 2006). Contudo, em cultivares suscetiveis, doses reduzidas de imazethapyr + imazapyr $\left(5,9+2,0 \mathrm{~g} \mathrm{ha}^{-1}\right)$ ocasionaram redução no crescimento, que levou a perdas na produtividade do arroz (Bond et al., 2006).

A massa seca da parte aérea foi reduzida com o incremento na dose dos herbicidas estudados, exceto para o cultivar IRGA 422CL no segundo experimento, em que ela não foi afetada pelo herbicida nicosulfuron (Figuras 7 e 8). O efeito negativo foi mais evidente no cultivar IRGA 417, que apresentou menor produção de massa seca, comparativamente ao cultivar IRGA 422CL.

Para o IRGA 422CL, em aplicação realizada no estádio $V_{2}$, o imazethapyr + imazapic causou redução no acúmulo de massa seca da parte aérea superior à do nicosulfuron (Figura 7). No cultivar IRGA 417, maior redução também foi observada com imazethapyr + imazapic, o que fica evidenciado na inflexão das curvas, que representam maiores reduções em menores doses deste herbicida (Figuras 7 e 8). Resultados semelhantes foram reportados por $\mathrm{Dal}$ Magro (2006), onde se observa que a produção de massa seca da parte aérea foi reduzida com o aumento na dose de imazethapyr + imazapic aplicada em cultivares suscetiveis. Em cultivares tolerantes, a redução no acúmulo de massa fresca da parte aérea também é crescente com a dose de imazethapyr, mas com recuperação das plantas durante o período de avaliação (Levy Jr. et al., 2006).

Esses resultados mostram que o cultivar IRGA 417, mesmo sendo suscetivel, pode tolerar doses reduzidas de nicosulfuron. E o cultivar IRGA 422CL pode sofrer injúrias com maiores doses de imazethapyr + imazapic.

A área foliar foi reduzida com o incremento na dose dos herbicidas, com maiores reduções observadas no cultivar IRGA 417 comparado a IRGA 422CL (Figuras 7 e 8). Ainda, nas menores doses dos herbicidas, o cultivar IRGA 417 produziu maior área foliar com nicosulfuron, comparativamente ao imazethapyr + imazapic; isso sugere menor eficácia deste herbicida para as espécies do gênero Oryza. Nas maiores doses testadas, o cultivar IRGA 422CL mostrou maior tolerância ao nicosulfuron.

Com relação às avaliações de massa seca da parte aérea e área foliar, é necessário enfatizar que elas foram realizadas aos 28 DAT, quando as plantas do cultivar IRGA 422CL estavam em processo de recuperação; portanto, esses dados não refletem a fitotoxicidade máxima que foi observada aos 21 DAT.

Os resultados dos dois estudos realizados mostraram que a tolerância apresentada pelo cultivar IRGA 422CL não está restrita à mistura formulada de imazethapyr + imazapic, mas ocorre também para o herbicida nicosulfuron. A tolerância dos cultivares de arroz depende da dose utilizada; incrementos nas doses dos herbicidas resultaram em respostas crescentes de fitotoxicidade ao arroz, afetando negativamente a estatura, massa seca da parte aérea e área foliar das plantas. Por outro lado, o cultivar IRGA 417 mostrou ser suscetivel à ação dos herbicidas estudados. 

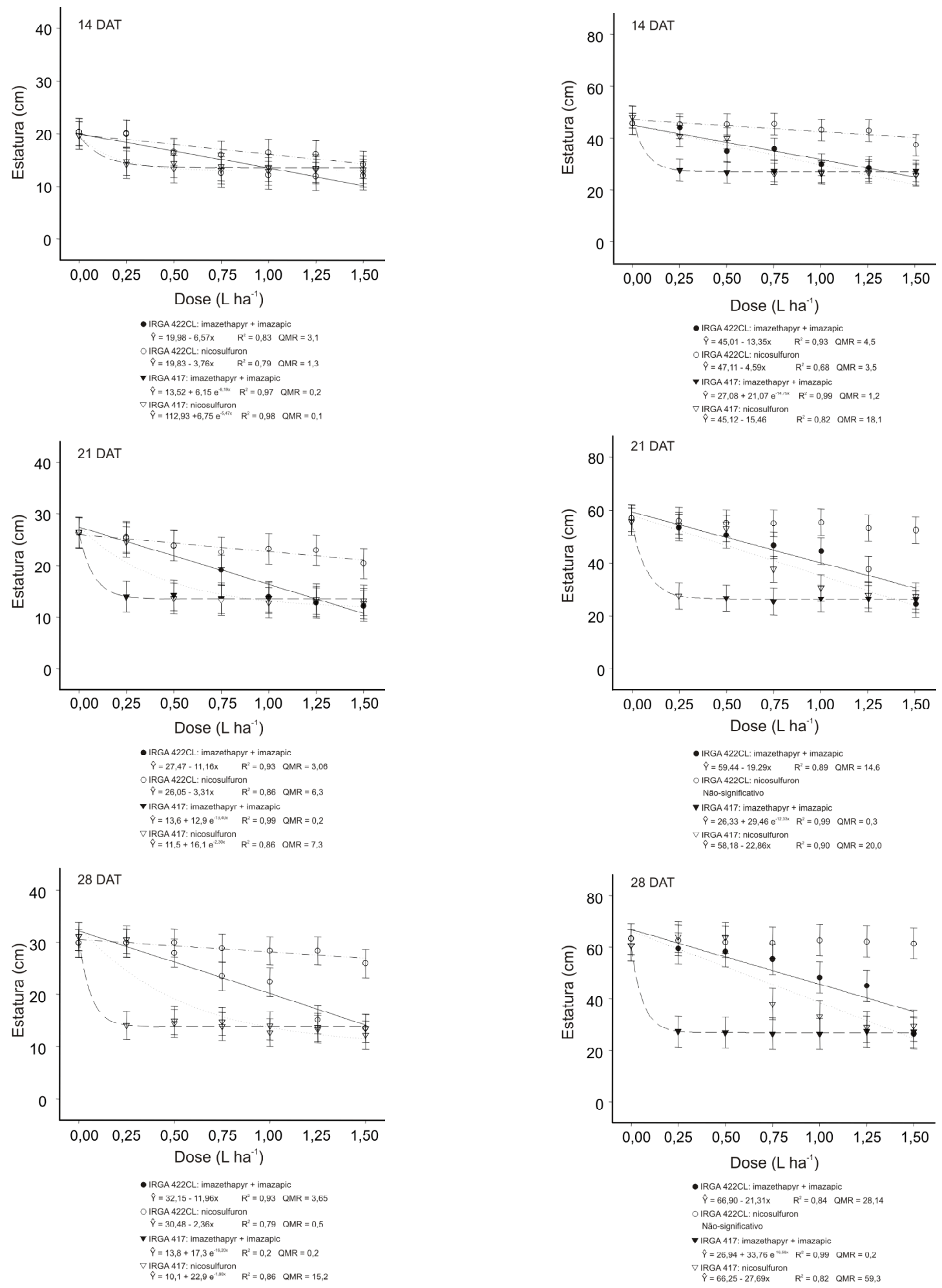

Figura 5 - Estatura de plantas de arroz irrigado em função da aplicação dos herbicidas Sanson ${ }^{\circledR}$ (40 $\mathrm{g} \mathrm{L}^{-1}$ de nicosulfuron) e Only ${ }^{\mathbb{B}}\left(75+25 \mathrm{~g} \mathrm{~L}^{-1}\right.$ de imazethapyr + imazapic), realizada no estádio $\mathrm{V}_{2}$. FAEM/UFPel, Capão do Leão/RS, 2005/06 (DAT: dias após o tratamento).

Figura 6 - Estatura de plantas de arroz irrigado em função da aplicação dos herbicidas Sanson ${ }^{\circledR}$ (40 $\mathrm{g} \mathrm{L}^{-1}$ de nicosulfuron) e Only ${ }^{\mathbb{B}}\left(75+25 \mathrm{~g} \mathrm{~L}^{-1}\right.$ de imazethapyr + imazapic), realizada no estádio $\mathrm{V}_{4}$. FAEM/UFPel, Capão do Leão/RS, 2005/06 (DAT: dias após o tratamento). 

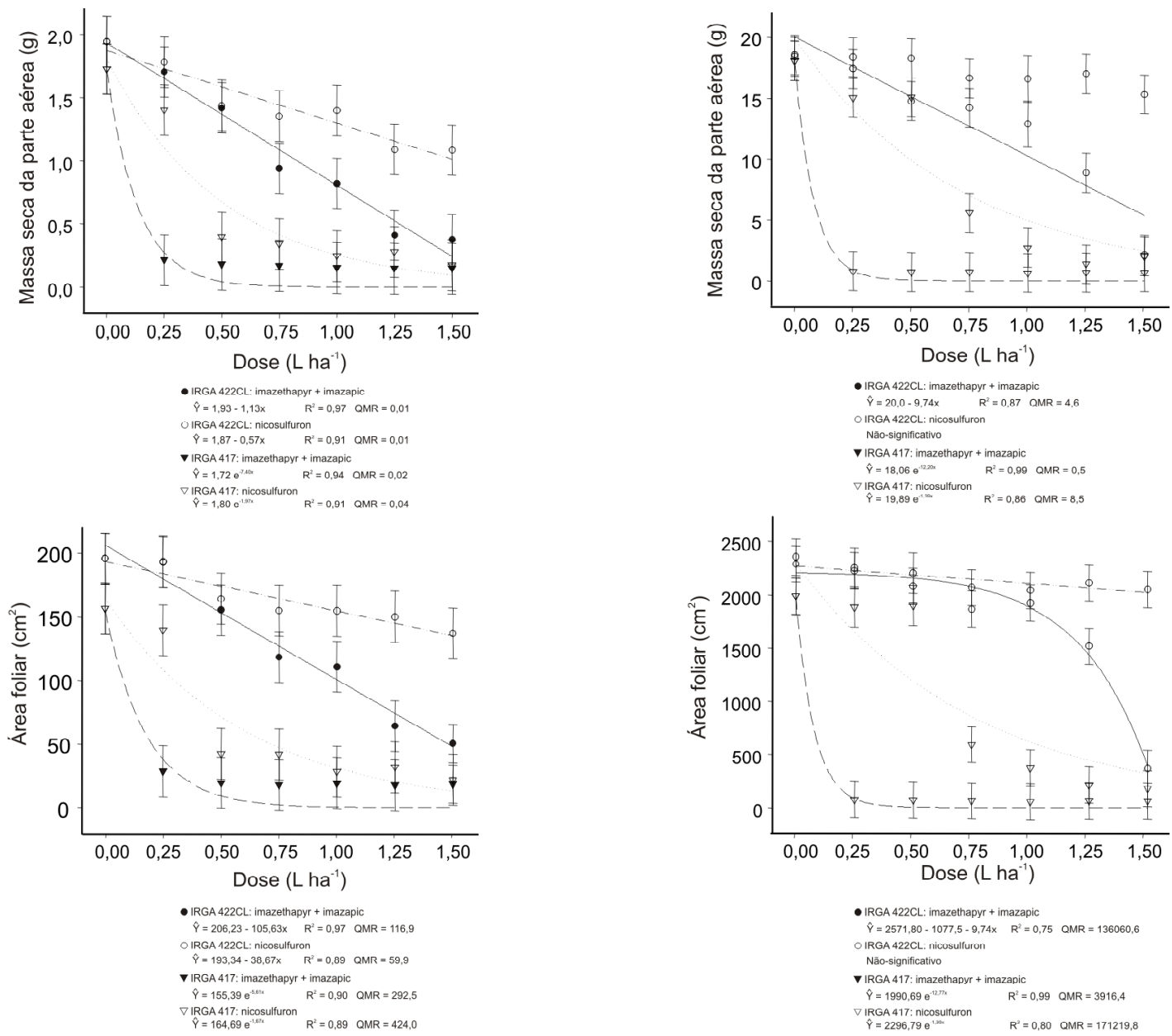

Figura 7 - Massa seca da parte aérea e área foliar de plantas de arroz irrigado em função da aplicação dos herbicidas Sanson ${ }^{\circledR}$ (40 $\mathrm{g} \mathrm{L}^{-1}$ de nicosulfuron) e Only ${ }^{\circledR}\left(75+25 \mathrm{~g} \mathrm{~L}^{-1} \mathrm{de}\right.$ imazethapyr + imazapic), realizada no estádio $\mathrm{V}_{2}$, avaliadas aos 28 dias após o tratamento. FAEM/UFPel, Capão do Leão/RS, 2005/06.

\section{LITERATURA CITADA}

ANDREI, E. Compêndio de defensivos agrícolas. 7.ed. São Paulo: Andrei, 2005. 1141 p.

AVILA, L. A. et al. Effect of flood timing on red rice (Oryza spp.) control with imazethapyr applied at different dryseeded rice growth stages. Weed Technol., v. 19, n. 2, p. $479-480,2005$.

BOND, J. A. et al. Corn and rice response to simulated drift of imazethapyr plus imazapyr. Weed Technol., v. 20, n. 1, p. $113-117,2006$.

COUNCE, P. A.; KEISLING, T. C.; MITCHELL, A. J. A uniform, objective, and adaptative system for expressing rice development. Crop Sci., v. 40, p. 436-443, 2000.

DAL MAGRO, T. Efeito de deriva simulada e época de aplicação do herbicida imazethapyr + imazapic no arroz irrigado (Oryza sativa L.). 2006. 65 f. Dissertação (Mestrado em Fitossanidade) - Universidade Federal de Pelotas, Pelotas, 2006.

DAL MAGRO, T. et al. Suscetibilidade de cultivares de arroz irrigado (Oryza sativa) à deriva simulada do herbicida imazethapyr + imazapic. Planta Daninha, v. 24, p. 751759, 2006.

ELLIS, J. M. et al. Rice (Oryza sativa) and corn (Zea mays) response to simulated drift of glyphosate and glufosinate. Weed Technol., v. 17, p. 452-460, 2003. 
EMPRESA BRASILEIRA DE PESQUISA AGROPECUÁRIA - EMBRAPA. Centro Nacional de Pesquisa de Solos. Sistema brasileiro de classificação de solos. Rio de Janeiro: 1999. 412 p.

KURTZ, M. E.; STREET, J. E. Response of rice (Oryza sativa) to glyphosate applied to simulate drift. Weed Technol., v. 17, p. 234-238, 2003.

LEVY Jr., R. J. et al. Effect of cultural practices on weed control and crop response in imidazolinone-tolerant rice. Weed Technol., v. 20, p. 249-254, 2006.

LOPES, M. C. B. et al. IRGA 422CL: A nova cultivar desenvolvida pelo programa de melhoramento genético do Instituto Rio Grandense do Arroz para o Sistema de Produção Clearfield ${ }^{\circledR}$. In: CONGRESSO BRASILEIRO DE ARROZ IRRIGADO, 3.; REUNIÃO DA CULTURA DO ARROZ IRRIGADO, 25., 2003, Balneário Camboriú. Anais... Itajaí: EPAGRI, 2003. p. 3-5.

OTTIS, B. V.; CHANDLER, J. M.; McCAULEY, G. N Imazethapyr application methods and sequences for imidazolinone-tolerant rice. Weed Technol., v. 17, p. 526533, 2003.
PELLERIN, K. J.; WEBSTER, E. P. Imazethapyr at different rates and timings in drill- and water-seeded imidazolinone-tolerant rice. Weed Technol., v. 18, p. 223227, 2004.

SOCIEDADE SUL-BRASILEIRA DE ARROZ IRRIGADO - SOSBAI. Arroz irrigado: recomendações técnicas da pesquisa para o Sul do Brasil. Santa Maria: 2005. 159 p.

STEELE, G. L.; CHANDLER, J. M.; MCCAULEY, G. N. Control of red rice (Oryza sativa) in imidazolinona-tolerant rice (Oryza sativa). Weed Technol., v. 16, p. 627-63 0, 2002.

TREZZI, M. M.; VIDAL, R. A. Herbicidas inibidores da ALS. In: VIDAL, R. A.; TREZZI, M. M. Herbicidologia, Porto Alegre, 2001. p. 25-36.

VILLA, S. C. C. et al. Controle de arroz-vermelho em dois genótipos de arroz (Oryza sativa) tolerantes a herbicidas do grupo das imidazolinonas. Planta Daninha, v. 24, p. 549$555,2006$.

WEBSTER, E. P.; MASSON, J. A. Acetolactate synthaseinhibiting herbicides on imidazolinona-tolerant rice. Weed Sci., v. 49, p. 652-657, 2001. 